\title{
Mitochondrial DNA copy number is reduced in male combat veterans with PTSD
}

Francesco Saverio Bersani (1, 2), Claire Morley (1), Daniel Lindqvist (1, 3), Elissa S. Epel (1, 4), Martin Picard (5), Rachel Yehuda (6), Janine Flory (6), Linda M. Bierer (6), Iouri Makotkine (6), Duna Abu-Amara (7), Michelle Coy (1), Victor I. Reus (1), Jue Lin (8), Elizabeth H. Blackburn (8), Charles Marmar (7), Owen M. Wolkowitz (1), Synthia H. Mellon (9)

1. Department of Psychiatry, University of California San Francisco, San Francisco, CA, USA

2. Department of Neurology and Psychiatry, Sapienza University of Rome, Rome, Italy

3. Department of Clinical Sciences, Section for Psychiatry, Lund University, Lund, Sweden

4. Center for Health and Community, University of California San Francisco, San Francisco, CA, USA

5. Center for Mitochondrial and Epigenomic Medicine, The Children's Hospital of Philadelphia and University of Pennsylvania, Philadelphia, Pennsylvania, USA

6. Department of Psychiatry, MSSM/James J. Peters Veterans Administration Medical Center, New York, NY, USA

7. Department of Psychiatry, Steven and Alexandra Cohen Veterans Center for Posttraumatic Stress and Traumatic Brain Injury, New York, NY, USA

8. Department of Biochemistry and Biophysics, University of California San Francisco, San Francisco, CA, USA

9. Department of $O B / G Y N$ and Reproductive Science, University of California San Francisco, San Francisco, CA, USA

\section{Corresponding author}

Owen Wolkowitz, MD

401 Parnassus Ave, Box F-0984

San Francisco, CA 94143-0984

Tel: (415) 476-7433 Fax: (415) 502-2661

E-mail: Owen.Wolkowitz@ucsf.edu 


\section{ABSTRACT}

Introduction: Mitochondrial abnormalities may be involved in PTSD, although few studies have examined this. Mitochondrial DNA copy number (mtDNAcn) in blood cells is an emerging systemic index of mitochondrial biogenesis and function. The present study assessed mtDNAcn in male combat-exposed veterans with PTSD compared to those without PTSD as well as its correlation with clinical scales.

Methods: mtDNAcn was assessed with a TaqMan multiplex assay in granulocytes of 43 male combat veterans with $(n=43)$ or without $(n=44)$ PTSD. Twenty of the PTSD subjects had co-morbid major depressive disorder (MDD). The Clinician Administered PTSD Scale (CAPS), the Positive and Negative Affect Schedule (PANAS), the Early Trauma Inventory (ETI) and the Beck Depression Inventory II (BDI-II) were used for the clinical assessments. All analyses were corrected for age and BMI.

Results: mtDNAcn was significantly lower in subjects with PTSD ( $p<0.05)$. Within the PTSD group, those with moderate PTSD symptom severity had relatively higher mtDNAcn than those with mild or severe symptoms $(\mathrm{p}<0.01)$. Within the PTSD group, mtDNAcn was positively correlated with PANAS positive subscale ratings $(\mathrm{p}<0.01)$ but was not significantly correlated with PANAS negative subscale, ETI or BDI-II ratings.

Discussion: This study provides the first evidence of: (i) a significant decrease of mtDNAcn in combat PTSD, (ii) a possible "inverted-U” shaped relationship between PTSD symptom severity and mtDNAcn within PTSD subjects, and (iii) a direct correlation of mtDNAcn with positive affectivity within PTSD subjects. Altered mtDNAcn in PTSD may reflect impaired energy metabolism, which might represent a novel aspect of its pathophysiology.

Key Words: Post-traumatic stress disorder, PTSD, mitochondria, mitochondrial DNA, positive affect, war veterans 


\section{INTRODUCTION}

Post-traumatic stress disorder (PTSD) is a debilitating mental illness characterized by recurrent distressing memories of an initial traumatic event, emotional numbing and hyperarousal (APA 2013). In addition to the traditional psychiatric symptoms, individuals with PTSD have a substantially higher medical burden, with increased rates of cardiometabolic disturbances and early mortality, suggesting widespread physical and biological concomitants of the disease (Levine et al. 2014).

Such physical and biological concomitants of PTSD may, in part, result from significant dysfunctions in several cellular processes including alterations in inflammation (Lindqvist et al. 2014, Wilson et al. 2013), oxidative stress (Ceprnja et al. 2011, Wilson et al. 2013), telomere homeostasis (Jergovic et al. 2014, O'Donovan et al. 2011), neuroendocrine regulation (Rasmusson et al. 2010, Yehuda 2001), nitric oxide (NO) production (Bersani et al. 2015, Lopez-Figueroa et al. 1998, Yeh et al. 2002) and mitochondrial activity (Li et al. 2014, Mellon et al. 2015, Su et al. 2008). Mitochondria are ubiquitous organelles of eukaryotic systems (Johannsen and Ravussin 2009, Scheffler 2001). They can be considered the "power generators" of the cell, converting oxygen, energy substrates (carbohydrates and lipids) and other compounds via oxidative phosphorylation into adenosine triphosphate (ATP), which is then transported to the cell's cytoplasm to fuel the majority of cellular reactions (Johannsen and Ravussin 2009, Scheffler 2001). Beyond energy production, mitochondria also play key roles in other crucial cellular processes such as apoptotic and necrotic cell death (Johannsen and Ravussin 2009, Kasahara and Scorrano 2014), regulation of gene expression (Johannsen and Ravussin 2009, Kasahara and Scorrano 2014, Picard et al. 2014b), and signal transduction for cell proliferation and differentiation (Johannsen and Ravussin 2009, Kasahara and Scorrano 2014). As a result, mitochondrial impairments are associated with, and may lead to, widespread psychiatric and somatic illnesses, with brain, heart and metabolism being especially vulnerable (Marazziti 2012, Dimauro and Davidzon 2005).

Cells of different tissues contain multiple mitochondria, and, in turn, each mitochondrion contains multiple copies of its own genome - the mitochondrial DNA (mtDNA) - which encodes 37 genes essential to energy production (Campbell et al. 2012, Clay Montier et al. 2009). One way of indirectly assessing mitochondrial function is by measuring the number of mtDNA molecules per cell, namely mitochondrial DNA copy number (mtDNAcn). MtDNAcn is also matched to cellular energy needs. High-energy requiring cells, such as heart, skeletal muscles and neurons require large quantities of ATP and maintain high numbers of mtDNA copies while low-energy requiring cells, such as spleen and endothelial cells, maintain fewer copies (Moyes et al 1998). Certain brain 
regions also contain varying amounts of mitochondria (Fuke et al., 2011). In addition to baseline differences, changes in mtDNA can reflect variations in mitochondrial biogenesis (i.e. the formation of new mitochondria). Exercise increases cellular energy demand and, as a compensatory responses, enhances energy production capacity through mitochondrial biogenesis, which can be detected as an increased mtDNAcn in various brain regions and other tissues (Steiner et al. 2011). On the other hand, mitochondrial content in skeletal muscle tends to decrease in sedentary individuals with aging, and this is reflected by a lower mtDNAcn (Short et al. 2005). MtDNAcn is therefore considered a marker of mitochondrial energetic function (Moyes et al. 1998, Clay Montier et al. 2009) and biogenesis (Lee HC and Wei YH 2005, Clay Montier et al. 2009).

While the exact meaning/significance of mtDNAcn is still not completely known, the importance of mtDNAcn maintenance for cell physiology and homeostasis is underscored by the evidence of altered mtDNAcn associated with diverse types of human disease, including developmental delays in early childhood (Macmillan and Shoubridge 1996), multiple sclerosis (Blokhin et al. 2008), renal and breast cancers (Xing et al. 2008, Yu et al. 2007), liver disease (Morten et al. 2007), biliary atresia (Tiao et al. 2007), type 2 diabetes (Choi et al. 2001, Lee H. K. et al. 1998), insulin resistance (Choi et al. 2001, Lee H. K. et al. 1998), cardiomyopathy (Bai R. K. and Wong 2005) and metabolic syndrome (Kim J. H. et al. 2012). Also, certain genetic defects that impair mitochondrial biogenesis can lead to depletion of mtDNA, which dramatically reduces mitochondrial energy production capacity leading to multisystemic disease (Al-Hussaini 2014; Hudson and Chinnery 2006). However, excessive levels of mtDNAcn can also be, less commonly, associated with disease (Clay Montier et al. 2009). This highlights the importance of appropriate regulation of a "just right" level of mtDNAcn for adaptive cellular physiology (Clay Montier et al. 2009).

A small number of studies also examined mtDNAcn (primarily in leukocytes) in relation to psychiatric disturbances, showing conflicting results. The mtDNAcn has been reported to be significantly high in autism (Giulivi et al. 2010), significantly reduced in some (but not in all) studies on bipolar disorder (Chang et al. 2014, de Sousa et al. 2014, Kakiuchi et al. 2005, Sabunciyan et al. 2007, Torrell et al. 2013, Vawter et al. 2006) and positively associated with childhood adversity and lifetime psychopathology in healthy people (Tyrka et al. 2015). A recent study in community-dwelling women $(n=142)$ found significantly lower mtDNAcn in people with higher depressive symptoms (not necessarily diagnosed with Major Depressive Disorder [MDD]) compared to those with lower depressive symptoms (Kim M. Y. et al. 2011). However, one study found that mtDNAcn was not significantly different in subjects with MDD compared to controls (He Y. et al. 2014). 
Although mtDNAcn has never previously been examined in PTSD, a small number of studies has examined mitochondrial function in this condition. In a post-mortem study, $\mathrm{Su}$ et al. investigated gene expression profiles in the dorsolateral-prefrontal cortex (DLPFC) of subjects with PTSD vs controls ( $\mathrm{Su}$ et al. 2008). They found that, of the 119 dysregulated genes in PTSD subjects, large clusters of functionally-related genes were associated with mitochondrial dysfunction (4.8\%) and oxidative phosphorylation (3.8\%) ( $\mathrm{Su}$ et al. 2008). $\mathrm{Li}$ et al found altered expression of mitochondrial genes in the amygdala of an animal model of PTSD (Li et al. 2014). A metabolomic study from our research team found PTSD subjects to have significantly altered plasma concentrations of several metabolites, consistent with mitochondrial dysfunction (i.e. altered Krebs cycle metabolites, carnitines and ketone bodies) (Mellon et al. 2015).

There is a need to study mitochondria in an accessible way in well-characterized PTSD samples, so that we may better understand how mitochondrial abnormalities may be related to the pathophysiology of the disease. In this research, we assessed granulocyte mtDNAcn in a sample of male war veterans with PTSD in comparison with psychiatrically healthy male war veterans without PTSD (i.e. controls). We hypothesized that mtDNAcn would be decreased in PTSD and would be inversely associated with the severity of clinical ratings.

\section{METHODS}

\subsection{Ethical statement}

The Institutional Review Boards of Icahn School of Medicine at Mount Sinai (ISMMS; New York, NY), the James J. Peters Veterans Administration Medical Center (JJPVAMC; Bronx, New York), New York University Medical Center (NYU; New York, NY), and the University of California, San Francisco, Medical Center (UCSF; San Francisco, CA) approved this study. Study participants gave written and informed consent to participate. The study was conducted in accordance with the provisions of the Helsinki Declaration.

\subsection{Recruitment procedures and Study participants}

Eighty-seven Operation Iraqi Freedom (OIF) and Operation Enduring Freedom (OEF) male combat-exposed veterans participated. Forty-three of the participants were diagnosed with current PTSD, while 44 did not have PTSD (i.e. controls). Among the PTSD subjects, 20 were diagnosed with concurrent MDD. Participants were recruited by NYU, ISMMS and JJPVAMC. Subjects were recruited from the Mental Health Services of the Manhattan, Bronx and Brooklyn Veterans Affairs Medical Centers, other regional VA medical centers, Veterans Service Organizations, National 
Guard, reservist agencies and organizations and from the general community. Recruitment methods included flyers, in-person presentations, media advertisements, internet postings (e.g. Craigslist) and referral from clinicians. Participants were compensated for their participation. Criteria for inclusion were: (a) PTSD subjects were positive for the presence of current combat-related PTSD of at least 3 months duration, as defined by the DSM-IV (First 1997), and the Clinician Administered PTSD Scale (CAPS) (Blake et al. 1990) criteria with a current CAPS score $>40$ (one PTSD subject met DSM-IV criteria for PTSD but was missing CAPS data; he was included in the study); (b) control subjects were also combat-exposed but were negative for lifetime PTSD and had a current CAPS score <20; (c) age between 20 and 60; (d) males; and (e) proficient in the English language. The following exclusion criteria were employed for all subjects: (a) history of alcohol dependence within the past 8 months; (b) history of drug abuse or dependence (except nicotine dependence) within the past year; (c) lifetime history of any psychiatric disorder with psychotic features, bipolar disorder, or obsessive-compulsive disorder; (d) those who were currently exposed to recurrent trauma or have been exposed to a traumatic event within the past 3 months; (e) subjects with prominent suicidal or homicidal ideation; (f) neurologic disorder or systemic illness affecting central nervous system function; ( $\mathrm{g}$ ) history of anemia, recent blood donation in the past 2 months; (i) subjects on medication who were not stable for $2+$ months on psychiatric medication, anticonvulsants, antihypertensive medication or sympathomimetic medication; (j) subjects who were classified with a moderate or severe traumatic brain injury (TBI) on the Ohio State University TBI Identification Method-Short Form; and finally (k) subjects who experienced loss of consciousness for greater than $10 \mathrm{~min}$. All study participants experienced combat traumas described in criterion A of DSM-IV PTSD diagnostic criteria. Structured Clinical Interviews for DSM-IV disorders (SCID) (First 1997) were conducted by doctoral level psychologists, and were audio recorded and calibrated weekly with a senior clinician in the PTSD program.

\subsection{Psychiatric and psychological assessment measures}

The SCID was used to determine whether participants met DSM-IV diagnostic criteria for any psychiatric disorder (First 1997). The CAPS was used to determine the severity of current PTSD symptoms (past month; "CAPS current") and the severity of the most severe lifetime episode of combat-related PTSD ("CAPS lifetime") (Blake et al. 1990). The Positive and Negative Affect Schedule (PANAS) was used to assess the positive and negative affectivity (Watson et al. 1988). Depression symptom severity was assessed with the self-rated Beck Depression Inventory-II (BDIII) (Beck et al. 1996). Exposure to early life trauma was evaluated using the Early Trauma Inventory (ETI)-Self Report Short Form (Bremner et al. 2007). 


\subsection{Blood samples and measurement of mtDNA copy number}

Blood was drawn in the morning after a night of fasting. Whole blood was collected into $10 \mathrm{ml}$ EDTA Lavender Top (LTT) tubes and granulocytes were isolated and stored at -80C. Peripheral blood mononuclear cells (PBMCs) were purified whole blood using standard Ficoll gradient centrifugation method. Granulocytes were prepared from the red blood pellets after Ficoll separation of the PBMCs by lysing in three volumes of ACK lysis buffer (QIAgen, cat \#158902). The cells were left in ACK lysis buffer at room temperature for 10 minutes with inversion every 2 minutes. The cells were spun at $400 \mathrm{~g}$ for 10 minutes in a Sorvall Legend RT tabletop centrifuge at $10^{\circ} \mathrm{C}$. The cell pellets were washed twice with $10 \mathrm{ml}$ of cold DPBS (Invitrogen, cat \# 14040-133) and spun at $400 \mathrm{~g}$ for 10 minutes at $10^{\circ} \mathrm{C}$. After the second wash, the cell pellets were resuspended in $5 \mathrm{ml}$ of DPBS, aliquoted into 5 of $1.5 \mathrm{ml}$ Eppendorf tubes, spun at $7000 \mathrm{rpm}$ for 5 minutes at $4^{\circ} \mathrm{C}$, and were stored at $-80^{\circ} \mathrm{C}$ for batch DNA purification. DNA was purified using QIAamp blood mini kit (cat\# 51106) based on the manufacture's manual and quantity were assessed with a nanodrop spectrophotometer. Relative copy number of mtDNA per diploid nuclear genome was determined using a TaqMan multiplex assay by the detection of a $69 \mathrm{bp}$ fragment of the ND1 gene in mtDNA (nucleotides 3485-3553) and an 87 bp fragment of RNase P (TaqMan® Copy Number Reference Assay, human, RNase P, cat\# 4403328, Life Technologies) in the nuclear genomic DNA (nDNA). This assay was adapted from previous published methods (He L. et al. 2002). The primer and probe sequences for ND1 are: ND1-forward [5'-CCCTAAAACCCGCCACATCT-3'], ND1-reverse [5'GAGCGATGGTGAGAGCTAAGGT-3'], ND1-FAM probe [5' CCATCACCCTCTACATCACCGCCC-TAMRA-3']. The reaction contained $12.5 \mathrm{ng}$ of total cellular (nuclear plus mitochondrial) genomic DNA, 100nM of ND1 probe, $300 \mathrm{nM}$ each of ND1forward primer and ND1-reverse primer each, 1X RNase P copy number Reference Assay, 1X LightCycler® 480 Probe Master (Roche, cat\# 04902343001) in a 10 ul reaction. All samples were run in triplicate wells in 384-well plates in a Roche LightCyler 480 . PCR condition is $95^{\circ} \mathrm{C} 10 \mathrm{~min}$ for 1 cycle; 45 cycles of $95{ }^{\circ} \mathrm{C} 10 \mathrm{sec}, 60^{\circ} \mathrm{C} 30 \mathrm{sec}, 72^{\circ} \mathrm{C} 1 \mathrm{sec}$ with data acquisition at $72^{\circ} \mathrm{C}$. Crossing point (Cp) for each well is derived by the LightCycler 480 program using the second derivation method. Relative copy number per diploid genome (i.e. per cell) is calculated by the following formula: Relative mtDNAcn=POWER $\{2,(\mathrm{CpND} 1-\mathrm{CpRNaseP})\}^{*} 2$. Inter-assay CV is $3.4 \%$ and intra-assay $\mathrm{CV}$ is $0.5 \%$.

\subsection{Statistical analysis}


The Statistical Package for the Social Sciences (SPSS) was used for statistical calculations. All tests were 2-tailed with an alpha=0.05. Significance values between 0.05 and 0.10 are reported as trends. Data are expressed as means \pm SD. The mtDNAcn across groups was not normally distributed and thus was transformed into normality using the Blom transformation, a statistical procedure replacing each raw score with its rank value and adjusting the scale distances between the ranks to achieve a normal distribution (Blom 1958); all other data were normally distributed. The MannWhitney U-test for continuous variables or the chi-square test for dichotomous variables was used to examine participants' baseline between-group differences. One-way analysis of covariance (ANCOVA) adjusting for age and body mass index (BMI), for their known association with mtDNA (Bratic and Larsson 2013, Lee J. Y. et al. 2014), was used to test for mtDNA intergroup differences. ANCOVA's were also performed to determine whether PTSD subjects with or without comorbid MDD, or with or without current antidepressant use, had significantly different mtDNAcn. Within PTSD subjects, Pearson partial correlation and quadratic regression using the same covariates (i.e. age and BMI) were used to determine associations between mtDNAcn and the clinical assessment scales.

\section{RESULTS}

\subsection{Demographics and clinical characteristics of the sample}

Demographic and clinical characteristics of the subjects are presented in Table 1. There were no significant differences with regard to age, years of education and smoking status. Hispanic ethnicity was more frequently represented in the PTSD sample. In addition, the amount of time since the index combat event was significantly longer in PTSD subjects. The use of antidepressant drugs was more common in the PTSD group $(\mathrm{p}<0.001)$, likely due to the PTSD group having cases of comorbid MDD and due to the use of antidepressants as a treatment for PTSD (Albucher and Liberzon 2002). However, the PTSD and control groups did not significantly differ in their use of other medications (e.g., statins, non-steroidal anti-inflammatory drugs (NSAIDs), anti-diabetic drugs). Subjects with concomitant somatic disorders (clinical hypertension, stable angina, prostate cancer and diabetes) were equally distributed across groups. As expected, subjects with PTSD compared to controls had significantly or near significantly higher scores on BDI-II $(\mathrm{p}<0.001)$, PANAS negative subscale $(\mathrm{p}<0.001)$, ETI $(\mathrm{p}=0.084)$, CAPS current $(\mathrm{p}<0.001)$ and CAPS lifetime $(\mathrm{p}<0.001)$ symptom severity scales, and significantly lower scores on PANAS positive subscale $(\mathrm{p}<0.001)$. 


\subsection{Intergroup differences in mtDNA copy number}

The mean mtDNAcn per cell in the PTSD group was $186.027 \pm 63.875$, compared to $207.785 \pm$ 72.329 in the control group (Figure 1). One-way ANCOVA (covarying for age and BMI) determined significant differences in mean mtDNAcn $(F(1,82)=4.24, p=0.043)$ between the two groups. The mtDNAcn did not significantly differ between PTSD subjects with (183.143 \pm 67.289$)$ or without $(188.536 \pm 62.126)$ comorbid MDD ( $\mathrm{p}=0.746)$, or between PTSD subjects who did $(190.539 \pm 80.244)$ or did not $(184.891 \pm 56.288)$ use antidepressants $(\mathrm{p}=0.128)$.

\subsection{Association of mtDNA copy number with clinical measures within PTSD sample}

To explore the relationship between PTSD symptom severity and mtDNAcn within the PTSD group, PTSD subjects were divided in tertiles according to ratings on the CAPS current scale. The mtDNAcn was $159.402 \pm 51.668$ for the "mild PTSD" subgroup ( $\mathrm{n}=14$; CAPS current score range: 44-56), $231.239 \pm 81.528$ for the "moderate PTSD” subgroup ( $n=14$; CAPS current score range: 57 $75)$ and $168.586 \pm 23.515$ for the "severe PTSD" subgroup ( $n=14$; CAPS current score range: 76100) (Figure 2). Age, BMI, number of individuals with concomitant MDD diagnosis and number of individuals using antidepressants were not significantly different between the three sub-groups ( $p>0.15$ ). One-way ANCOVA (covarying for age and BMI) determined significant differences in mean mtDNAcn $(\mathrm{F}(2,37)=6.479, \mathrm{p}=0.004)$ between the three subgroups. Post-hoc Bonferroni testing revealed that subjects with moderate PTSD had significantly higher mtDNAcn than subjects with either mild $(\mathrm{p}=0.006)$ or severe $(\mathrm{p}=0.018)$ PTSD, but did not differ significantly from controls $(\mathrm{p}=1.000)$.

The relationship between CAPS current ratings and mtDNAcn did not fit a linear correlation ( $\mathrm{r}=-$ 0.027, $\mathrm{p}=0.869)$ but, rather, approximated a curvilinear quadratic correlation $(\mathrm{r}=0.363, \mathrm{p}=0.064)$. On the other hand, mtDNAcn was significantly positively correlated with PANAS positive affect subscale in a linear manner $(\mathrm{r}=0.437 ; \mathrm{p}=0.005)$ (Figure 3$)$ but not with PANAS negative affect subscale ( $r=0.218 ; p=0.177)$, ETI ( $r=-0.030 ; p=0.856)$, CAPS lifetime ( $r=0.052 ; p=0.750)$ or BDI-II $(\mathrm{r}=0.053 ; \mathrm{p}=0.744)$. Lastly, mtDNAcn was not significantly correlated with time since the index combat event within the PTSD group $(\mathrm{r}=0.228, \mathrm{p}=0.163)$.

\section{DISCUSSION}

This is the first study assessing the mtDNAcn, a widely used marker of mitochondrial function and biogenesis (Clay Montier et al. 2009), in individuals with PTSD and its correlation with clinical measures. Our results showed (i) a significantly lower granulocyte mtDNAcn in combat-exposed 
male PTSD subjects compared to combat-exposed male controls without PTSD (Figure 1), (ii) an inverted-U-shaped relationship between mtDNAcn and PTSD severity (i.e., subjects with moderate current PTSD had relatively higher mtDNAcn that subjects with either mild or severe current PTSD) (Figure 2), and (iii) a significant direct correlation between mtDNAcn and positive affectivity within PTSD subjects (Figures 3). The mean mtDNAcn was not different between PTSD subjects with or without co-morbid MDD as well as between PTSD subjects taking or not taking antidepressants, suggesting that the observed decrease in mtDNAcn in PTSD is likely not attributable to comorbid MDD diagnosis or antidepressant use.

Even though some previous clinical studies have shown alterations in mtDNAcn in various psychiatric disorders including autism, MDD and bipolar disorder (Chang et al. 2014, Giulivi et al. 2010, Kim M. Y. et al. 2011), this is the first study to investigate mtDNAcn in PTSD. A small number of clinical or pre-clinical studies (reviewed in the Introduction) previously implicated mitochondrial dysfunction in the pathophysiology of PTSD (Li et al. 2014, Mellon et al. 2015, Su et al. 2008). However, our study differs from those by studying a clinical sample rather than an animal model and by using in vivo blood-based, rather than post mortem brain-based, mitochondrial markers.

Several mechanisms might help explain the decreased mtDNAcn in PTSD. At the cellular level, mtDNAcn is tightly regulated (Bai Y. et al. 2000, Cao et al. 2007, Clay Montier et al. 2009), with (i) low ("sub-threshold") mtDNAcn generally triggering an up-regulation of mtDNA replication and high ("supra-threshold") mtDNAcn generally triggering mtDNA degradation (Clay Montier et al. 2009), and (ii) specific mtDNA regulatory proteins contributing to the maintenance of mtDNAcn within a physiologic range (Clay Montier et al. 2009, Campbell et al 2012). Our data showing lower mtDNAcn in individuals with PTSD raise the possibility that PTSD is characterized by dysregulated mtDNAcn thresholds and/or maintenance, with significantly increased degradation or decreased replication of mtDNA in comparison to controls. Our finding of decreased mtDNAcn in PTSD is also consistent with the findings of $\mathrm{Su}$ et al ( $\mathrm{Su}$ et al. 2008) and Li et al (Li et al. 2014) showing that a high number of genes related to mitochondrial function may be dysregulated in PTSD or animal models of PTSD, although the specific cellular effects of these dysregulations are not completely known.

At the neurobiological level, PTSD has been associated with down-regulation of glucocorticoid and serotonergic systems, as well as with reduced nitric oxide (NO) production, although conflicting results exist (Bersani et al. 2015, Bugajski 1999, Harris et al. 2000, Lopez-Figueroa et al. 1998, Meewisse et al. 2007, Persoons et al. 1995, Southwick et al. 1999, Yeh et al. 2002). Such downregulation may contribute to the association between PTSD and decreased mtDNAcn. First, both 
increased and decreased glucocorticoid activity can be detrimental for mitochondria via multiple changes in mtDNA gene expression and increased reactive oxygen species (ROS) (Du et al. 2009, Picard et al. 2014a, Psarra and Sekeris 2009, 2011, Tang et al. 2013). Second, in vitro studies (Garrett et al. 2014, Rasbach et al. 2010) show that agonists of 5-HT2 and 5-HT1f serotonergic receptors may increase mtDNAcn in parallel with PGC-1 $\alpha$ (peroxisome proliferator gamma coactivator $1 \alpha$ ), a potent inducer of mitochondrial biogenesis (Fernandez-Marcos and Auwerx 2011), suggesting a serotonergic-dependent mechanism controlling the expansion of mitochondrial content. Third, NO could act on mitochondria at several levels, and treatment of various cells with NO donors increases their mtDNA content (Kelly and Scarpulla 2004, Nisoli and Carruba 2006, Nisoli et al. 2003). Therefore, it is possible that down-regulated glucocorticoid, serotonergic or NO systems, which have been observed in some studies in PTSD (Bersani et al. 2015, Bugajski 1999, Harris et al. 2000, Lopez-Figueroa et al. 1998, Meewisse et al. 2007, Persoons et al. 1995, Southwick et al. 1999, Yeh et al. 2002), may directly or indirectly contribute to reduced mtDNA replication or, although less likely, increased mtDNA degradation.

In addition, several PTSD-associated phenotypes (e.g. metabolic dysregulation, increased BMI, visceral adiposity, diabetes, insulin resistance (Levine et al. 2014)) are themselves associated with lowered mtDNAcn (Choi et al. 2001, Kim J. H. et al. 2012, Lee H. K. et al. 1998, Lee J. Y. et al. 2014). However, we did not observe significant correlations between BMI and mtDNAcn in our samples.

At an organismal level, PTSD may be associated with aspects of accelerated biological aging (Bremner and Narayan 1998, Miller and Sadeh 2014, Moreno-Villanueva et al. 2013, Torgashov et al. 2013, Yehuda 2005), and decreased mtDNAcn could be further evidence of this (Bratic and Larsson 2013). One proposed index of accelerated biological aging in PTSD is premature telomere shortening (Jergovic et al. 2014, O'Donovan et al. 2011). Telomere length is genetically and epigenetically determined (Chan and Blackburn 2004) and is also subject to shortening by cellular stresses such as oxidation and inflammation (Chan and Blackburn 2004). Critically shortened telomeres elicit sustained DNA damage responses, such as activation of tumor suppressor protein p53 (Sahin et al. 2011), with outcomes that can include altered transcriptional program, cell cycle arrest, cellular senescence or apoptosis (Sahin et al. 2011). Activation of p53 can also decrease the expression of PGC-1a with subsequent decrease of mitochondrial function and mtDNAcn (Sahin et al. 2011).

Our second finding was an "inverted-U"-shaped relationship between PTSD symptom severity and mtDNAcn in the PTSD sample, although this was not hypothesized a priori and therefore requires replication. Specifically, we found that moderate levels of PTSD symptom severity were associated 
with relatively higher mtDNAcn compared to both low and high levels of symptom severity. There are several models by which mtDNAcn could show an "inverted-U" relationship with symptom severity. One model has been termed "mitochondrial allostatic load," in which conditions of mild cellular stress increase mitochondrial biogenesis and mtDNAcn, perhaps as a compensatory response, while higher or more chronic cellular stress leads to mitochondrial damage and dysfunction, perhaps due to exceeding compensatory capacity (Handschin and Spiegelman 2006, Moyes and Battersby 1998, Picard et al. 2014a, Scarpulla et al. 2012, Yu-Wai-Man et al. 2010). Therefore, the relative increase of mtDNAcn from mild to moderate PTSD severity could be explained by a compensatory adaptive increase of mtDNA proliferation, which may be driven by (i) the activation of PGC-1 $\alpha$ proteins (Handschin and Spiegelman 2006, Moyes and Battersby 1998, Scarpulla et al. 2012), and (ii) the translocation of telomerase reverse transcriptase (TERT; the catalytic subunit of telomerase) from the nucleus to the mitochondria with subsequent mitochondrial protection (Jaiswal et al. 2013, Saretzki 2009). On the other hand, the relative decrease of mtDNAcn from moderate to severe PTSD severity might be explained by evidence that, in the presence of high levels of cellular stressors, up to 80-90\% of TERT shuttles to the mitochondria (Ahmed et al. 2008), leading to insufficient nuclear telomere protection with subsequent telomere shortening cellular and mitochondrial damage (described above) (Ahmed et al. 2008, Saretzki 2009). These scenarios, however, remain purely hypothetical, but may be useful in guiding future research.

Our final major finding was the positive correlation of mtDNAcn with PANAS positive subscale ratings, but not with PANAS negative subscale, ETI or BDI-II depression ratings. This, too, was not hypothesized a priori; therefore it is also considered preliminary and in need of replication. The finding is, however, consistent with preliminary evidence of a significant positive correlation between mtDNAcn and PANAS positive subscale in individuals with MDD (Lindqvist et al. 2015).

\subsection{Strengths and limits}

Limitations of the present study include our use of an all male study sample; future studies, including an ongoing one by our group, will be needed to investigate biological parameters in combat-exposed females with or without PTSD. Since this was a cross-sectional study based on single time-point blood and behavioural measurements, we cannot determine moment-to-moment variability in the measures, and we cannot assess causal mediation models. Also, our study measured mtDNAcn in granulocytes; therefore, these results cannot uncritically be extrapolated to other cell types or tissues. While a strength of our study is our use of a combat-exposed non-PTSD group, since the non-specific effects of combat exposure are controlled for, we recognize that 
individuals who did not develop PTSD despite combat exposure may represent especially resilient individuals rather that normative control subjects. Also, we did not have diagnostic data on subsyndromal depression or other anxiety disorders besides obsessive-compulsive disorder, and the possible impact of these variables is not known. Finally, we are not proposing mtDNAcn as a biomarker for PTSD, both because we do not have data regarding its specificity for PTSD, and because our subjects with moderate PTSD did not differ from controls in mtDNAcn.

Strengths of the present study are that the sample was clinically well-characterized, information on potential covariates was available (medical illness, BMI, comorbid depression, medication, cigarette usage, time since index trauma event, etc.), all blood samples were drawn fasting and at the same time of day, and all assays were conducted in the same lab. An additional strength of the present study is its sample of relatively young war veterans, since age-related illnesses can pose significant confounds in studies of psychiatric disorders in older subjects and in studies of mtDNA (MengelFrom et al. 2014).

\subsection{Conclusion}

The present study provides the first evidence that the granulocyte mtDNAcn is decreased in combat-exposed men with PTSD, has a possible "inverted-U" shaped relationship with PTSD symptom severity, and is positively associated with positive affectivity. These findings add to the accumulating evidence that mitochondrial dysfunction is associated with, and may underlie, some of the psychiatric, physical and biological manifestations of PTSD, and contribute to an expanded view of PTSD, as being - in addition to a mental illness - an illness with important somatic underpinnings. If these findings are replicated in studies with larger samples and longitudinal designs, assessment of mtDNAcn could help clarify aspects of the pathophysiology of PTSD and could point to novel markers and treatment targets.

\section{Acknowledgements}

This study was supported by the following grants: U.S. Department of Defense, W81XWH-11-20223 (PI: Charles Marmar); U.S. Department of Defense, W81XWH-10-1-0021 (PI: Owen M. Wolkowitz); The Mental Illness Research, Education and Clinical Center (MIRECC). Daniel Lindqvist received financial support from the Sjobring Foundation; the OM Persson Foundation and the province of Scania (Sweden) state grants (ALF). The authors declare no conflict of interest. This publication arises from collaborative activities among eight institutions under the U.S. Department of Defense contract "Systems Biology Studies of PTSD": University of California San Francisco, New York University, Icahn School of Medicine at Mt. Sinai, US Army Medical Command 
(MEDCOM), University of California Santa Barbara, Institute for Systems Biology, Emory University and the Veterans Administration Health Care System. 
Table 1. Demographic and clinical characteristics of PTSD subjects and controls

\begin{tabular}{|c|c|c|c|c|}
\hline & $\begin{array}{l}\text { PTSD } \\
\text { N: } 43\end{array}$ & $\begin{array}{c}\text { Controls } \\
\text { N: } 44\end{array}$ & $\begin{array}{c}\text { Mann-Whitney } \\
U \text {-test }\end{array}$ & $X^{2}$ \\
\hline Age (years, mean \pm SD) & $34.49 \pm 8.15$ & $32.16 \pm 8.55$ & 0.075 & \\
\hline Years of education $($ mean \pm SD) & $12.86 \pm 3.90$ & $13.20 \pm 5.39$ & 0.107 & \\
\hline Gender & All males & All males & & \\
\hline Smokers (n) & 7 & 3 & & 0.161 \\
\hline BMI (mean \pm SD) & $30.34 \pm 5.55$ & $28.08 \pm 4.25$ & 0.070 & \\
\hline Hispanic/ Non-Hispanic (n) & $23 / 19$ & $15 / 29$ & & $0.054 *$ \\
\hline Time since index combat event (months, mean \pm SD) & $68.68 \pm 25.75$ & $51.89 \pm 24.99$ & $0.003 *$ & \\
\hline \multicolumn{5}{|l|}{ Medications } \\
\hline Taking statins (n) & 2 & 1 & & 0.572 \\
\hline Taking antidepressants (n) & 14 & 1 & & $<0.001 *$ \\
\hline Taking NSAIDs (n) & 3 & 2 & & 0.625 \\
\hline Taking antidiabetic drugs (n) & 1 & 0 & & 0.321 \\
\hline \multicolumn{5}{|l|}{ Comorbid diseases } \\
\hline Clinical hypertension (n) & 5 & 4 & & 0.794 \\
\hline Stable angina $(\mathrm{n})$ & 2 & 1 & & 0.463 \\
\hline Diabetes (n) & 3 & 0 & & 0.122 \\
\hline Prostate cancer (n) & 0 & 1 & & 0.320 \\
\hline \multicolumn{5}{|l|}{ Clinical measures } \\
\hline CAPS total current $($ mean \pm SD) & $68.52 \pm 16.34$ & $3.77 \pm 5.47$ & $<0.001 *$ & \\
\hline CAPS total lifetime (mean \pm SD) & $93.45 \pm 13.55$ & $9.09 \pm 8.57$ & $<0.001 *$ & \\
\hline BDI-II (mean \pm SD) & $23.50 \pm 10.39$ & $5.72 \pm 6.77$ & $<0.001 *$ & \\
\hline ETI (mean \pm SD) & $8.19 \pm 5.85$ & $5.91 \pm 4.35$ & 0.084 & \\
\hline PANAS positive (mean \pm SD) & $25.31 \pm 7.29$ & $33.84 \pm 9.59$ & $<0.001 *$ & \\
\hline PANAS negative (mean \pm SD) & $28.57 \pm 8.60$ & $15.23 \pm 5.30$ & $<0.001 *$ & \\
\hline MDD diagnosis (n) & 20 & 0 & & $<0.001 *$ \\
\hline
\end{tabular}

$* p \leq 0.05$ 
Figure 1. Scatterplots of mtDNA copy number in PTSD subjects vs controls. ANCOVA: $(F(1,82)=4.239, p=0.043)$

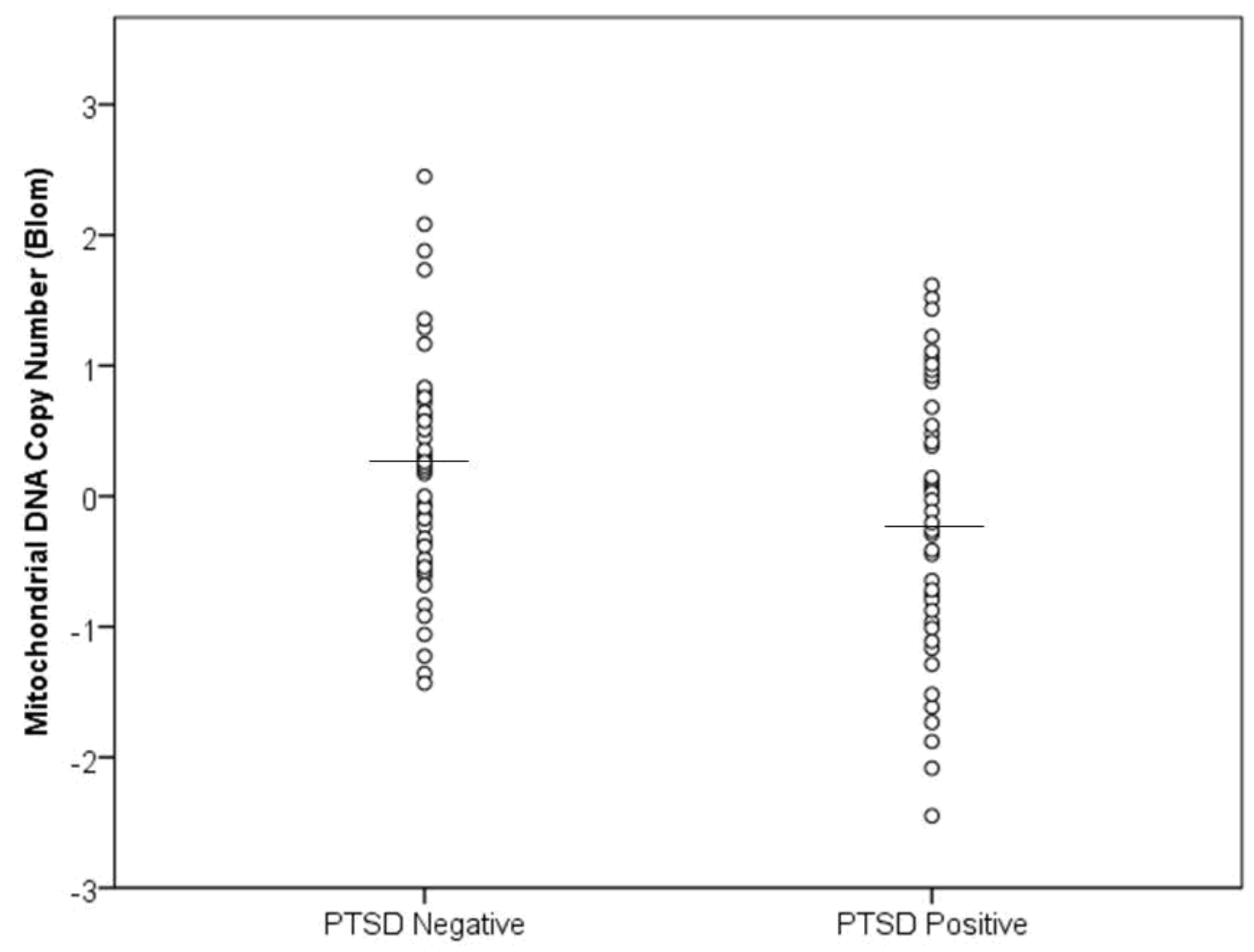


Figure 2. mtDNA copy number in mild, moderate and severe PTSD subjects. ANCOVA: $F(2,37)=6.479, p=0.004$ $* p<0.05$ at the Bonferroni post-hoc test

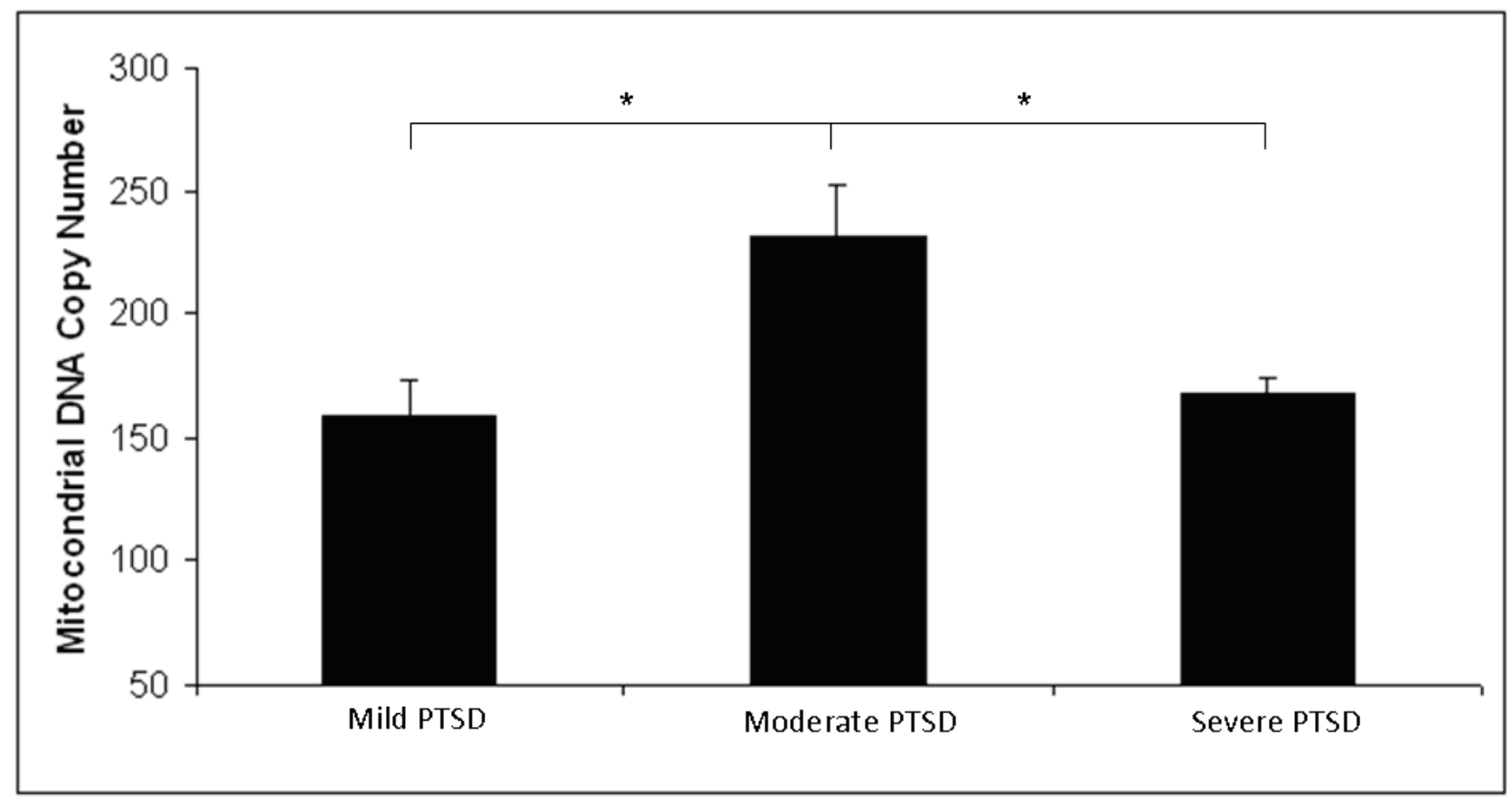


Figure 3. Scatterplot of the correlation between mtDNA copy number and PANAS positive subscale within PTSD subjects $(r=0.437 ; p=0.005)$

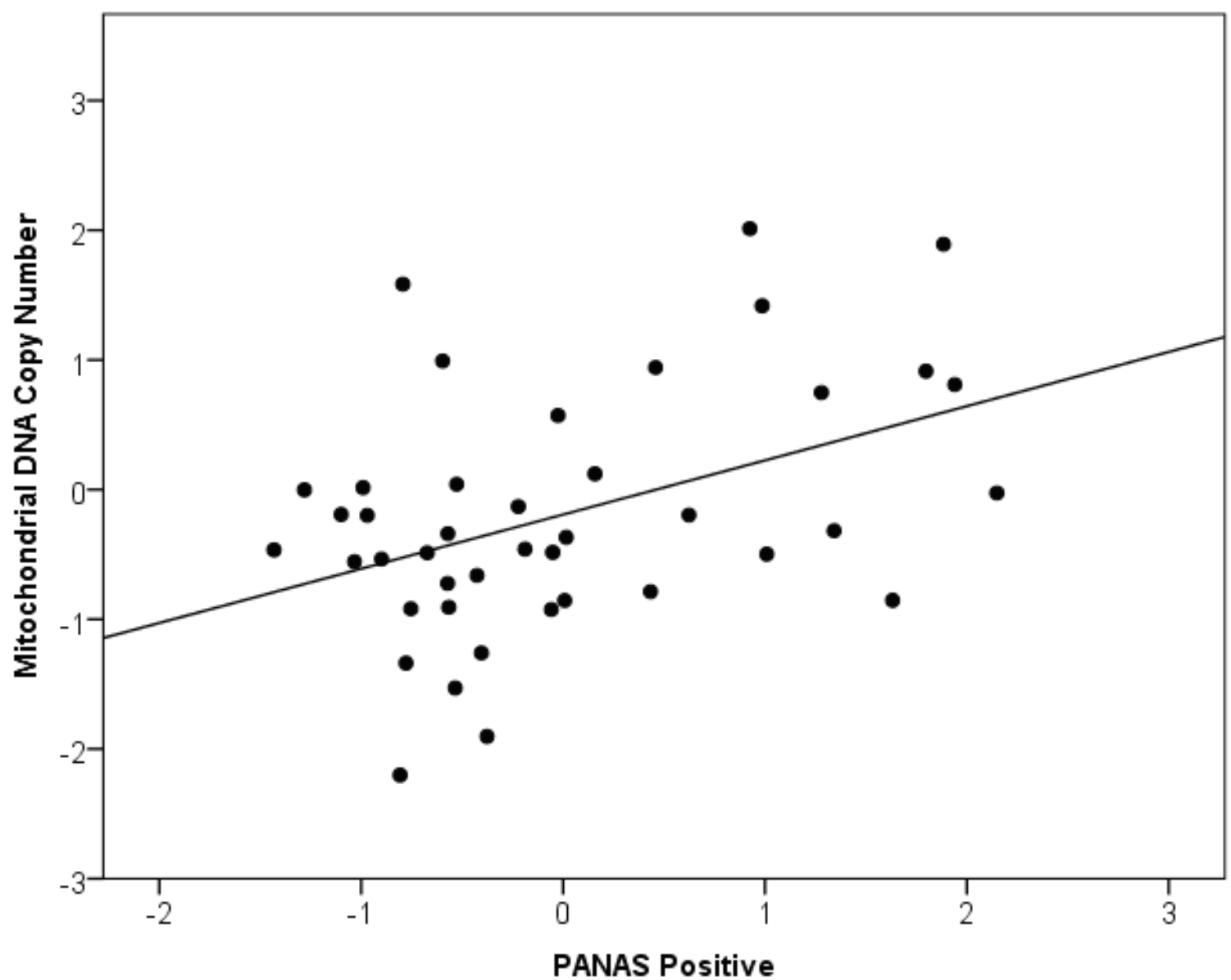




\section{REFERENCES}

Al-Hussaini A et al. 2014. Clinical and molecular characteristics of mitochondrial DNA depletion syndrome associated with neonatal cholestasis and liver failure. J Pediatr 164: 553-559

America Psychiatric Association 2013. Diagnostic and statistical manual of mental disorders (5th ed.). Washington: American Psychiatric Association.

Ahmed S, Passos JF, Birket MJ, Beckmann T, Brings S, Peters H, Birch-Machin MA, von Zglinicki T, Saretzki G. 2008. Telomerase does not counteract telomere shortening but protects mitochondrial function under oxidative stress. J Cell Sci 121: 1046-1053.

Al-Kafaji G, Golbahar J. 2013. High glucose-induced oxidative stress increases the copy number of mitochondrial DNA in human mesangial cells. Biomed Res Int 2013: 754946.

Albucher RC, Liberzon I. 2002. Psychopharmacological treatment in PTSD: a critical review. J Psychiatr Res 36: 355-367.

Bai RK, Wong LJ. 2005. Simultaneous detection and quantification of mitochondrial DNA deletion(s), depletion, and over-replication in patients with mitochondrial disease. J Mol Diagn 7: 613-622.

Bai Y, Shakeley RM, Attardi G. 2000. Tight control of respiration by NADH dehydrogenase ND5 subunit gene expression in mouse mitochondria. Mol Cell Biol 20: 805-815.

Beck AT, Steer RA, G.K. B. 1996. Manual for the Beck Depression Inventory-II: Psychological Corporation, San Antonio, TX.

Bersani FS, et al. 2015. Nitric Oxide synthetic capacity is decreased in PTSD: Correlations with PTSD symptom severity, negative affect and telomerase activity. Abstract Book of the 2015 Annual Meeting of Society of Biological Psychiatry. Biol Psychiatry 77: 367

Blake DD, Weathers FW, Nagy LM, Kaloupek DG, Klauminzer G, Charney DS, Keane TM. 1990. A clinician rating scale for assessing current and lifetime PTSD: the CAPS-1. Behav Ther 13: 187-188.

Blokhin A, Vyshkina T, Komoly S, Kalman B. 2008. Variations in mitochondrial DNA copy numbers in MS brains. J Mol Neurosci 35: 283-287.

Blom G. 1958. Statistical Estimates and Transformed Beta Variables. New York: John Wiley \& Sons.

Bratic A, Larsson NG. 2013. The role of mitochondria in aging. J Clin Invest 123: 951-957.

Bremner JD, Narayan M. 1998. The effects of stress on memory and the hippocampus throughout the life cycle: implications for childhood development and aging. Dev Psychopathol 10: 871-885.

Bremner JD, Bolus R, Mayer EA. 2007. Psychometric properties of the Early Trauma Inventory-Self Report. J Nerv Ment Dis 195: 211-218.

Bugajski J. 1999. Social stress adapts signaling pathways involved in stimulation of the hypothalamicpituitary-adrenal axis. J Physiol Pharmacol 50: 367-379.

Campbell CT, Kolesar JE, Kaufman BA. 2012. Mitochondrial transcription factor A regulates mitochondrial transcription initiation, DNA packaging, and genome copy number. Biochim Biophys Acta 1819: 921-929.

Cao L, Shitara H, Horii T, Nagao Y, Imai H, Abe K, Hara T, Hayashi J, Yonekawa H. 2007. The mitochondrial bottleneck occurs without reduction of mtDNA content in female mouse germ cells. Nat Genet 39: 386-390. 
Ceprnja M, Derek L, Unic A, Blazev M, Fistonic M, Kozaric-Kovacic D, Franic M, Romic Z. 2011. Oxidative stress markers in patients with post-traumatic stress disorder. Coll Antropol 35: 1155-1160.

Chan SR, Blackburn EH. 2004. Telomeres and telomerase. Philos Trans R Soc Lond B Biol Sci 359: 109121.

Chang CC, Jou SH, Lin TT, Liu CS. 2014. Mitochondrial DNA variation and increased oxidative damage in euthymic patients with bipolar disorder. Psychiatry Clin Neurosci 68: 551-557.

Choi YS, Kim S, Pak YK. 2001. Mitochondrial transcription factor A (mtTFA) and diabetes. Diabetes Res Clin Pract 54 Suppl 2: S3-9.

Clay Montier LL, Deng JJ, Bai Y. 2009. Number matters: control of mammalian mitochondrial DNA copy number. J Genet Genomics 36: 125-131.

de Sousa RT, Uno M, Zanetti MV, Shinjo SM, Busatto GF, Gattaz WF, Marie SK, Machado-Vieira R. 2014. Leukocyte mitochondrial DNA copy number in bipolar disorder. Prog Neuropsychopharmacol Biol Psychiatry 48: 32-35.

Dimauro S, Davidzon G. 2005. Mitochondrial DNA and disease. Ann Med 37: 222-232.

Du J, McEwen B, Manji HK. 2009. Glucocorticoid receptors modulate mitochondrial function: A novel mechanism for neuroprotection. Commun Integr Biol 2: 350-352.

Fernandez-Marcos PJ, Auwerx J. 2011. Regulation of PGC-1alpha, a nodal regulator of mitochondrial biogenesis. Am J Clin Nutr 93: 884S-890.

First MB. 1997. Structured Clinical Interview for DSM-IV axis I disorders. Washington: American Psychiatric Press

Fuke S, Kubota-Sakashita M, Kasahara T, Shigeyoshi Y, Kato T. 2011. Regional variation in mitochondrial DNA copy number in mouse brain. Biochimica Biophysica Acta 1807: 270-274.

Garrett SM, Whitaker RM, Beeson CC, Schnellmann RG. 2014. Agonism of the 5-hydroxytryptamine 1F receptor promotes mitochondrial biogenesis and recovery from acute kidney injury. J Pharmacol Exp Ther 350: $257-264$.

Giulivi C, Zhang YF, Omanska-Klusek A, Ross-Inta C, Wong S, Hertz-Picciotto I, Tassone F, Pessah IN. 2010. Mitochondrial dysfunction in autism. JAMA 304: 2389-2396.

Handschin C, Spiegelman BM. 2006. Peroxisome proliferator-activated receptor gamma coactivator 1 coactivators, energy homeostasis, and metabolism. Endocr Rev 27: 728-735.

Harris CW, Edwards JL, Baruch A, Riley WA, Pusser BE, Rejeski WJ, Herrington DM. 2000. Effects of mental stress on brachial artery flow-mediated vasodilation in healthy normal individuals. Am Heart J 139: 405-411.

He L, Chinnery PF, Durham SE, Blakely EL, Wardell TM, Borthwick GM, Taylor RW, Turnbull DM. 2002. Detection and quantification of mitochondrial DNA deletions in individual cells by real-time PCR. Nucleic Acids Res 30: e68.

He Y, Tang J, Li Z, Li H, Liao Y, Tang Y, Tan L, Chen J, Xia K, Chen X. 2014. Leukocyte mitochondrial DNA copy number in blood is not associated with major depressive disorder in young adults. PLoS One 9: e96869. 
Hudson G and Chinnery PF. 2006. Mitochondrial DNA polymerase-gamma and human disease. Hum Mol Genet 15 Spec No 2: 244-252

Jaiswal RK, Kumar P, Yadava PK. 2013. Telomerase and its extracurricular activities. Cell Mol Biol Lett 18: 538-554.

Jergovic M, et al. 2014. Telomere shortening and immune activity in war veterans with posttraumatic stress disorder. Prog Neuropsychopharmacol Biol Psychiatry 54: 275-283.

Johannsen DL, Ravussin E. 2009. The role of mitochondria in health and disease. Curr Opin Pharmacol 9: 780-786.

Kakiuchi C, Ishiwata M, Kametani M, Nelson C, Iwamoto K, Kato T. 2005. Quantitative analysis of mitochondrial DNA deletions in the brains of patients with bipolar disorder and schizophrenia. Int $\mathrm{J}$ Neuropsychopharmacol 8: 515-522.

Kasahara A, Scorrano L. 2014. Mitochondria: from cell death executioners to regulators of cell differentiation. Trends Cell Biol 24: 761-770.

Kelly DP, Scarpulla RC. 2004. Transcriptional regulatory circuits controlling mitochondrial biogenesis and function. Genes Dev 18: 357-368.

Kim JH, Im JA, Lee DC. 2012. The relationship between leukocyte mitochondrial DNA contents and metabolic syndrome in postmenopausal women. Menopause 19: 582-587.

Kim MY, Lee JW, Kang HC, Kim E, Lee DC. 2011. Leukocyte mitochondrial DNA (mtDNA) content is associated with depression in old women. Arch Gerontol Geriatr 53: e218-221.

Lee HC and Wei YH. 2005. Mitochondrial biogenesis and mitochondrial DNA maintenance of mammalian cells under oxidative stress. Int J Biochem Cell Biol. 37: 822-834

Lee HK, Song JH, Shin CS, Park DJ, Park KS, Lee KU, Koh CS. 1998. Decreased mitochondrial DNA content in peripheral blood precedes the development of non-insulin-dependent diabetes mellitus. Diabetes Res Clin Pract 42: 161-167.

Lee JY, Lee DC, Im JA, Lee JW. 2014. Mitochondrial DNA copy number in peripheral blood is independently associated with visceral fat accumulation in healthy young adults. Int J Endocrinol 2014: 586017.

Levine AB, Levine LM, Levine TB. 2014. Posttraumatic stress disorder and cardiometabolic disease. Cardiology 127: 1-19.

Li H, Li X, Smerin SE, Zhang L, Jia M, Xing G, Su YA, Wen J, Benedek D, Ursano R. 2014. Mitochondrial Gene Expression Profiles and Metabolic Pathways in the Amygdala Associated with Exaggerated Fear in an Animal Model of PTSD. Front Neurol 5: 164.

Liesa M and Shirihai OS. 2013. Mitochondrial dynamics in the regulation of nutrient utilization and energy expenditure. Cell Metab 17: 491-506

Lindqvist D, et al. 2015. Mitochondrial DNA copy number in MDD: A pilot study. Abstract Book of the 2015 Annual Meeting of Society of Biological Psychiatry. Biol Psychiatry 77: 545

Lindqvist D, et al., 2014. Proinflammatory milieu in combat-related PTSD is independent of depression and early life stress. Brain Behav Immun 42: 81-88. 
Lopez-Figueroa MO, Day HE, Akil H, Watson SJ. 1998. Nitric oxide in the stress axis. Histol Histopathol 13: 1243-1252.

Macmillan CJ, Shoubridge EA. 1996. Mitochondrial DNA depletion: prevalence in a pediatric population referred for neurologic evaluation. Pediatr Neurol 14: 203-210.

Marazziti D, Baroni S, Picchetti M, Landi P, Silvestri S, Vatteroni E, Catena Dell'Osso M. 2012. Psychiatric disorders and mitochondrial dysfunctions. Eur Rev Med Pharmacol Sci 16: 270-275.

Meewisse ML, Reitsma JB, de Vries GJ, Gersons BP, Olff M. 2007. Cortisol and post-traumatic stress disorder in adults: systematic review and meta-analysis. Br J Psychiatry 191: 387-392.

Mellon SH, et al. 2015. Mitochondrial Dysfunction and Defective Uptake of Dietary Nutrients in Subjects with Post Traumatic Stress Disorder Article submitted to Molecular Psychiatry.

Mengel-From J, Thinggaard M, Dalgard C, Kyvik KO, Christensen K, Christiansen L. 2014. Mitochondrial DNA copy number in peripheral blood cells declines with age and is associated with general health among elderly. Hum Genet 133: 1149-1159.

Miller MW, Sadeh N. 2014. Traumatic stress, oxidative stress and post-traumatic stress disorder: neurodegeneration and the accelerated-aging hypothesis. Mol Psychiatry 19: 1156-1162.

Moreno-Villanueva M, Morath J, Vanhooren V, Elbert T, Kolassa S, Libert C, Burkle A, Kolassa IT. 2013. $\mathrm{N}$-glycosylation profiling of plasma provides evidence for accelerated physiological aging in post-traumatic stress disorder. Transl Psychiatry 3: e320.

Morten KJ, et al. 2007. Liver mtDNA content increases during development: a comparison of methods and the importance of age- and tissue-specific controls for the diagnosis of mtDNA depletion. Mitochondrion 7: 386-395.

Moyes C, Battersby B, Leary SC 1998. Regulation of muscle mitochondrial design. J Exp Biol 201: 299-307.

Nisoli E, Carruba MO. 2006. Nitric oxide and mitochondrial biogenesis. J Cell Sci 119: 2855-2862.

Nisoli E, et al. 2003. Mitochondrial biogenesis in mammals: the role of endogenous nitric oxide. Science 299: 896-899.

O'Donovan A, Epel E, Lin J, Wolkowitz O, Cohen B, Maguen S, Metzler T, Lenoci M, Blackburn E, Neylan TC. 2011. Childhood trauma associated with short leukocyte telomere length in posttraumatic stress disorder. Biol Psychiatry 70: 465-471.

Persoons JH, Schornagel K, Breve J, Berkenbosch F, Kraal G. 1995. Acute stress affects cytokines and nitric oxide production by alveolar macrophages differently. Am J Respir Crit Care Med 152: 619-624.

Picard M, Juster RP, McEwen BS. 2014a. Mitochondrial allostatic load puts the 'gluc' back in glucocorticoids. Nat Rev Endocrinol 10: 303-310.

Picard M, et al. 2014b. Progressive increase in mtDNA 3243A $>$ G heteroplasmy causes abrupt transcriptional reprogramming. Proc Natl Acad Sci U S A 111: E4033-4042.

Psarra AM, Sekeris CE. 2009. Glucocorticoid receptors and other nuclear transcription factors in mitochondria and possible functions. Biochim Biophys Acta 1787: 431-436.

Psarra AM, Sekeris CE. 2011. Glucocorticoids induce mitochondrial gene transcription in HepG2 cells: role of the mitochondrial glucocorticoid receptor. Biochim Biophys Acta 1813: 1814-1821. 
Rasbach KA, Funk JA, Jayavelu T, Green PT, Schnellmann RG. 2010. 5-hydroxytryptamine receptor stimulation of mitochondrial biogenesis. J Pharmacol Exp Ther 332: 632-639.

Rasmusson AM, Schnurr PP, Zukowska Z, Scioli E, Forman DE. 2010. Adaptation to extreme stress: posttraumatic stress disorder, neuropeptide Y and metabolic syndrome. Exp Biol Med (Maywood) 235: 11501162.

Sabunciyan S, Kirches E, Krause G, Bogerts B, Mawrin C, Llenos IC, Weis S. 2007. Quantification of total mitochondrial DNA and mitochondrial common deletion in the frontal cortex of patients with schizophrenia and bipolar disorder. J Neural Transm 114: 665-674.

Sahin E, et al. 2011. Telomere dysfunction induces metabolic and mitochondrial compromise. Nature 470: 359-365.

Saretzki G. 2009. Telomerase, mitochondria and oxidative stress. Exp Gerontol 44: 485-492.

Scarpulla RC, Vega RB, Kelly DP. 2012. Transcriptional integration of mitochondrial biogenesis. Trends Endocrinol Metab 23: 459-466.

Scheffler IE. 2001. Mitochondria make a come back. Adv Drug Deliv Rev 49: 3-26.

Short KR, Bigelow ML, Kahl J, Singh R, Coenen-Schimke J, Raghavakaimal S, Nair KS. 2005. Decline in skeletal muscle mitochondrial function with aging in humans. PNAS 102: 5618-5623

Southwick SM, Paige S, Morgan CA, 3rd, Bremner JD, Krystal JH, Charney DS. 1999. Neurotransmitter alterations in PTSD: catecholamines and serotonin. Semin Clin Neuropsychiatry 4: 242-248.

Steiner JL, Murphy EA, McClellan JL, Carmichael MD, Davis JM. 2011. Exercise training increases mitochondrial biogenesis in the brain. Journal of Applied Physiology 111: 1066-1071

Su YA, et al. 2008. Dysregulated mitochondrial genes and networks with drug targets in postmortem brain of patients with posttraumatic stress disorder (PTSD) revealed by human mitochondria-focused cDNA microarrays. Int J Biol Sci 4: 223-235.

Tang VM, Young AH, Tan H, Beasley C, Wang JF. 2013. Glucocorticoids increase protein carbonylation and mitochondrial dysfunction. Horm Metab Res 45: 709-715.

Tiao MM, Lin TK, Kuo FY, Huang CC, Du YY, Chen CL, Chuang JH. 2007. Early stage of biliary atresia is associated with significant changes in 8-hydroxydeoxyguanosine and mitochondrial copy number. J Pediatr Gastroenterol Nutr 45: 329-334.

Torgashov MN, Miakotnykh VS, Pal'tsev AI. 2013. [Age-related aspects of the extent of lipid metabolism and post-traumatic stress disorders among veterans of modern warfare]. Adv Gerontol 26: 525-532.

Torrell H, Montana E, Abasolo N, Roig B, Gaviria AM, Vilella E, Martorell L. 2013. Mitochondrial DNA (mtDNA) in brain samples from patients with major psychiatric disorders: gene expression profiles, mtDNA content and presence of the mtDNA common deletion. Am J Med Genet B Neuropsychiatr Genet 162B: 213223.

Tyrka AR, Parade SH, Price LH, Kao HT, Porton B, Philip NS, Welch ES, Carpenter LL. 2015. Alterations of Mitochondrial DNA Copy Number and Telomere Length with Early Adversity and Psychopathology. Biological Psychiatry in press.

Vawter MP, et al. 2006. Mitochondrial-related gene expression changes are sensitive to agonal-pH state: implications for brain disorders. Mol Psychiatry 11: 615, 663-679. 
Watson D, Clark LA, Tellegen A. 1988. Development and validation of brief measures of positive and negative affect: the PANAS scales. J Pers Soc Psychol 54: 1063-1070.

Wilson CB, McLaughlin LD, Nair A, Ebenezer PJ, Dange R, Francis J. 2013. Inflammation and oxidative stress are elevated in the brain, blood, and adrenal glands during the progression of post-traumatic stress disorder in a predator exposure animal model. PLoS One 8: e76146.

Xing J, et al. 2008. Mitochondrial DNA content: its genetic heritability and association with renal cell carcinoma. J Natl Cancer Inst 100: 1104-1112.

Yeh CB, Leckman J, Wan FJ, Shiah IS, Lu RB. 2002. Characteristics of acute stress symptoms and nitric oxide concentration in young rescue workers in Taiwan. Psychiatry Res 112: 59-68.

Yehuda R. 2001. Biology of posttraumatic stress disorder. J Clin Psychiatry 62 Suppl 17: 41-46.

Yehuda R. 2005. Neuroendocrine aspects of PTSD. Handb Exp Pharmacol: 371-403.

Yu-Wai-Man P, Sitarz KS, Samuels DC, Griffiths PG, Reeve AK, Bindoff LA, Horvath R, Chinnery PF. 2010. OPA1 mutations cause cytochrome c oxidase deficiency due to loss of wild-type mtDNA molecules. Hum Mol Genet 19: 3043-3052.

Yu M, Zhou Y, Shi Y, Ning L, Yang Y, Wei X, Zhang N, Hao X, Niu R. 2007. Reduced mitochondrial DNA copy number is correlated with tumor progression and prognosis in Chinese breast cancer patients. IUBMB Life 59: 450-457. 